\title{
Large-amplitude electromagnetic ion cyclotron waves and density fluctuations in the flank of the earth's magnetosheath
}

\author{
J. S. Zhao, T. Y. Wang, M. W. Dunlop, C. Shi, J. S. He, \\ X. C. Dong, D. J. Wu, B. Giles and C. T. Russell
}

\section{Published version information}

Citation: JS Zhao et al. "Large-amplitude electromagnetic ion cyclotron waves and density fluctuations in the flank of the earth's magnetosheath." Geophysical Research Letters, vol. 46, no. 9 (2019): 4545-4553.

DOI: $\underline{10.1029 / 2019 G L 081964}$

This version is made available in accordance with publisher policies. Please cite only the published version using the reference above. This is the citation assigned by the publisher at the time of issuing the APV. Please check the publisher's website for any updates. 


\section{Geophysical Research Letters}

\author{
RESEARCH LETTER \\ 10.1029/2019GL081964 \\ Key Points: \\ - MMS observes a long-lasting \\ large-amplitude electromagnetic ion \\ cyclotron wave event in the \\ dawnside flank of the \\ magnetosheath \\ - Density fluctuations are both \\ positively and negatively correlated \\ with the parallel magnetic field \\ - Local excitation of the \\ electromagnetic ion cyclotron \\ instability and mirror instability \\ contributes to the observed \\ fluctuations
}

Supporting Information:

- Supporting Information S1

Correspondence to:

J. S. Zhao,

js_zhao@pmo.ac.cn

Citation:

Zhao, J. S., Wang, T. Y., Dunlop, M. W. Shi, C., He, J. S., Dong, X. C., et al. (2019). Large-amplitude

electromagnetic ion cyclotron waves and density fluctuations in the flank of the Earth's magnetosheath. Geophysical Research Letters, 46, 4545-4553. https:// doi.org/10.1029/2019GL081964

Received 8 JAN 2019 Accepted 11 APR 2019 Accepted article online 16 APR 2019 Published online 3 MAY 2019
(C)2019. American Geophysical Union. All Rights Reserved.

\section{Large-Amplitude Electromagnetic Ion Cyclotron Waves and Density Fluctuations in the Flank of the Earth's Magnetosheath}

\author{
J. S. Zhao' ${ }^{1}$, T. Y. Wang ${ }^{2}$ (D) M. W. Dunlop ${ }^{2,3}$ D, C. Shi ${ }^{1,4}$, J. S. He ${ }^{5}$, X. C. Dong ${ }^{3}$ D, D. J. Wu' \\ B. Giles ${ }^{6}$ iD, and C. T. Russell ${ }^{7}$ iD \\ ${ }^{1}$ Key Laboratory of Planetary Sciences, Purple Mountain Observatory, Chinese Academy of Sciences, Nanjing, China, \\ ${ }^{2}$ RAL Space, STFC, Oxfordshire, UK, ${ }^{3}$ School of Space and Environment, Beihang University, Beijing, China, ${ }^{4}$ College of \\ Astronautics, Nanjing University of Aeronautics and Astronautics, Nanjing, China, ${ }^{5}$ School of Earth and Space Sciences, \\ Peking University, Beijing, China, ${ }^{6}$ NASA Goddard Space Flight Center, Greenbelt, MD, USA, ${ }^{7}$ Department of Earth, \\ Planetary and Space Sciences, University of California, Los Angeles, CA, USA
}

\begin{abstract}
The electromagnetic ion cyclotron (EMIC) wave usually observed in the Earth's magnetosheath is thought to be generated through the ion temperature anisotropy instability. This paper presents an observation of a long-lasting large-amplitude EMIC wave event in the dawnside flank of the magnetosheath by the Magnetospheric Multiscale mission, lasting from 06:33:00 to 12:35:00 UT on 16 April 2018. The wave amplitude is around $0.2 \mathrm{nT}$ as compared to the ambient magnetic field $\sim 12 \mathrm{nT}$. The characteristic frequency and scale size are around $0.2 \mathrm{~Hz}$ and $1,028 \mathrm{~km}$, respectively. Accompanying EMIC waves are density fluctuations, which exhibit both positive and negative correlations with the longitudinal magnetic field. Using the fitted parameters for the ion and electron phase space densities, plasma kinetic theory predicts local excitations of both EMIC and mirror instabilities, which provide the energy of these observed EMIC waves and density fluctuations.
\end{abstract}

Plain Language Summary The Earth's Magnetosheath is a region slowing down high-speed particles originating from the solar wind, protecting the Earth from the hazards of high-energy charged particles. A considerable fraction of the energy of energetic particles is transferred into plasma waves. This paper presents an observational event of large-amplitude low-frequency electromagnetic waves lasting nearly $6 \mathrm{hr}$, propagating from the outer magnetosheath to the inner magnetosheath. Density fluctuations are found to coexist with these electromagnetic waves. Applying the measurements of charged particles to modeling, we show the observed waves are likely excited by free energy provided by ions with an anisotropic temperature distribution, consistent with wave theory.

\section{Introduction}

The Earth's magnetosheath is a region between the Earth's bow shock and magnetopause (Lucek et al., 2005). A considerable fraction of the plasma bulk flow energy in the solar wind is converted into the plasma thermal energy in the magnetosheath, where the plasma temperature perpendicular to the magnetic field $T_{\perp}$ is usually larger than the parallel temperature $T_{\|}$(Lucek et al., 2005; Sckopke et al., 1990). A large ion temperature anisotropy with $T_{\perp}>T_{\|}$can also be developed in the plasma depletion layer nearby the magnetopause (e.g., Anderson et al., 1991; Zwan \& Wolf, 1976). This temperature anisotropy can trigger the ion cyclotron anisotropy instability and the mirror instability, producing the electromagnetic ion cyclotron (EMIC) wave and the mirror wave (Gary, 1992; Gary et al., 1993). Under the assumptions of a uniform plasma and a biMaxwellian ion velocity distribution, the EMIC instability has a nonzero real frequency and maximum growth at propagation parallel to the background magnetic field $\mathrm{B}_{0}$, whereas the ion mirror instability has a zero real frequency and maximum growth rate at propagation oblique to $B_{0}(G a r y, 1993)$. These two wave modes are frequently observed in the magnetosheath (e.g., Anderson et al., 1994; Czaykowska et al., 1998; Denton et al., 1994; Hubert et al., 1989; Lacombe et al., 1992; Sckopke et al., 1990; Tsurutani et al., 1982, 2011), especially downstream of the quasi-perpendicular bow shock (e.g., Schwartz et al., 1996).

EMIC waves observed in the magnetosheath can have large amplitude. For example, shortly after crossing of the quasi-perpendicular shock, EMIC waves having amplitude $\sim 10 \mathrm{nT}$ were found by the Cluster satellites, 
propagating along the downstream magnetic field where $B_{0} \sim 90 \mathrm{nT}$ (Alexandrova et al., 2004). Cassini and Wind spacecraft measurements have shown the existence of large-amplitude EMIC waves in the duskside flank of the magnetosheath where the peak amplitude is $\sim 4 \mathrm{nT}$ and $B_{0} \sim 25 \mathrm{nT}$, and in the subsolar magnetosheath where the peak amplitude is about $14 \mathrm{nT}$ compared with $B_{0} \sim 55 \mathrm{nT}$ (Remya et al., 2014). Furthermore, a statistical analysis from AMPTE/IRM spacecraft proposed that EMIC waves contribute mainly to electromagnetic fluctuations downstream of the quasi-perpendicular low-beta shocks and the average relative amplitude $\delta B / B_{0}$ is around 0.08 (Czaykowska et al., 2001). Recently, Zhao et al. (2018) applied the NASA Magnetospheric Multiscale (MMS) mission's high temporal resolution plasma measurements to show modulation of ion and electron pitch angle distribution in the presence of large-amplitude EMIC waves. These large-amplitude EMIC waves may play important roles in triggering nonlinear instability and scattering of ions in the magnetosheath (i.e., Alexandrova et al., 2004; Kitamura et al., 2018; McKean et al., 1995).

In addition to large-amplitude EMIC waves, EMIC waves have also been reported to occur simultaneously with plasma density fluctuations (Lacombe et al., 1995). However, the mechanism responsible for these density fluctuations still remains unknown. Using data from the MMS mission, this paper presents observational evidences of the coexistence of both large-amplitude EMIC waves and density fluctuations. Through the analyses of the observed wave characteristics, in combination with the linear instability predictions from plasma kinetic theory, we propose that the EMIC instability is responsible for EMIC waves, and both EMIC and mirror instabilities contribute to density fluctuations.

\section{Data Set}

This study analyzes the wave activity from electromagnetic field and plasma data of the MMS mission. Magnetic field measurements by the FluxGate Magnetometer (FGM) provide the magnetic field data with the temporal resolution of 16 samples per second in survey mode and 128 samples per second in burst mode (Russell et al., 2016). We use the electric field data with a sample rate of $32 \mathrm{~Hz}$ from the Electric Double Probes (Ergun et al., 2016; Lindqvist et al., 2016). Plasma measurements by the Fast Plasma Investigation (FPI) provide plasma data with the cadence of $4.5 \mathrm{~s}$ in fast mode and the cadences $150 \mathrm{~ms}$ for ions and 30 ms for electrons in burst mode (Pollock et al., 2016). Since the Dual Ion Spectrometer in FPI is unable to discriminate protons from other kinds of ion species, we assume all ions detected by Dual Ion Spectrometer/FPI contributed by protons. The density and temperature of ion species of $\mathrm{He}^{+}, \mathrm{He}^{++}$, and $\mathrm{O}^{+}$are obtained from the Hot Plasma Composition Analyzer (Young et al., 2016).

\section{Plasma Overview and Wave Observations}

The MMS spacecraft detects an EMIC wave event during the interval of 06:33:00-12:35:00 UT on 16 April 2018. As illustrated in the MMS1 trajectory (Figures $1 \mathrm{~h}$ and 1i), the spacecraft is located in the dawnside flank region of the magnetosheath. It moves from $(-3.62,-20.52,6.31) R_{E}$ at 06:33:00 UT to $(-2.19$, $-22.79,7.09) R_{E}$ at 12:35:00 UT. During this interval, the interplanetary magnetic field in GSE coordinates is $\mathbf{B}_{\mathrm{GSE}} \approx(-1.8,-3.5,0.6) \mathrm{nT}$, indicating the event is located downstream of the quasi-perpendicular bow shock. An overview of plasma and magnetic field is presented in Figures 1a-1g. Both plasma and magnetic field stay almost stable. During the prolonged interval of 09:55:00-11:20:00 UT, the time-averaged plasma parameters are $\mathrm{n}_{\mathrm{i}} \simeq 28.1 \mathrm{~cm}^{-3}, \mathrm{~T}_{\mathrm{i}} \simeq 76.9 \mathrm{eV}, \mathrm{T}_{\mathrm{e}} \simeq 23.6 \mathrm{eV}, \mathrm{B}_{0} \simeq 11.5 \mathrm{nT}$, Alfvén speed $\mathrm{V}_{\mathrm{A}}=\mathrm{B}_{0} / \sqrt{\mu_{0} \mathrm{~m}_{\mathrm{i}} \mathrm{n}_{\mathrm{i}}} \bumpeq$ $47.2 \mathrm{~km} / \mathrm{s}$, and ion bulk flow speed $\mathbf{V}_{\mathrm{GSE}} \simeq(-206.0,-73.9,41.6) \mathrm{km} / \mathrm{s}$ with an angle of $\sim 22.7^{\circ}$ with respect to the mean magnetic field. As labeled by the two dashed vertical lines in Figures 1a-1g, the burst mode continually operates during 10:23:14-10:58:32 UT, which provides a high temporal resolution data set supporting detailed analysis of plasma fluctuations.

Figures 2a presents the power spectral densities (PSDs) of the magnetic field, ion and electron velocity, and ion and electron number density by using Welch's method (Welch, 1967). Before calculating these PSDs, magnetic field data in burst mode (128 samples per second) are smoothly averaged over a time interval of 1,000 s, which are used as the background magnetic field. We then transform magnetic field and velocity vector data from GSE coordinates to a mean-field aligned coordinate system where three axes are defined as $\mathbf{e}_{\perp \mathbf{1}}=\mathbf{e}_{\perp \mathbf{2}} \times \mathbf{e}_{\|}, \mathbf{e}_{\perp \mathbf{2}}=\mathbf{e}_{\|} \times \mathbf{R} / R$, and $\mathbf{e}_{\|}=\mathbf{B}_{\mathbf{0}} / B_{0}$ and $\mathbf{R}$ is the position vector of MMS1. Here and throughout this paper the terms "perpendicular" and "parallel" refer to directions with respect to $\mathrm{B}_{0}$. 

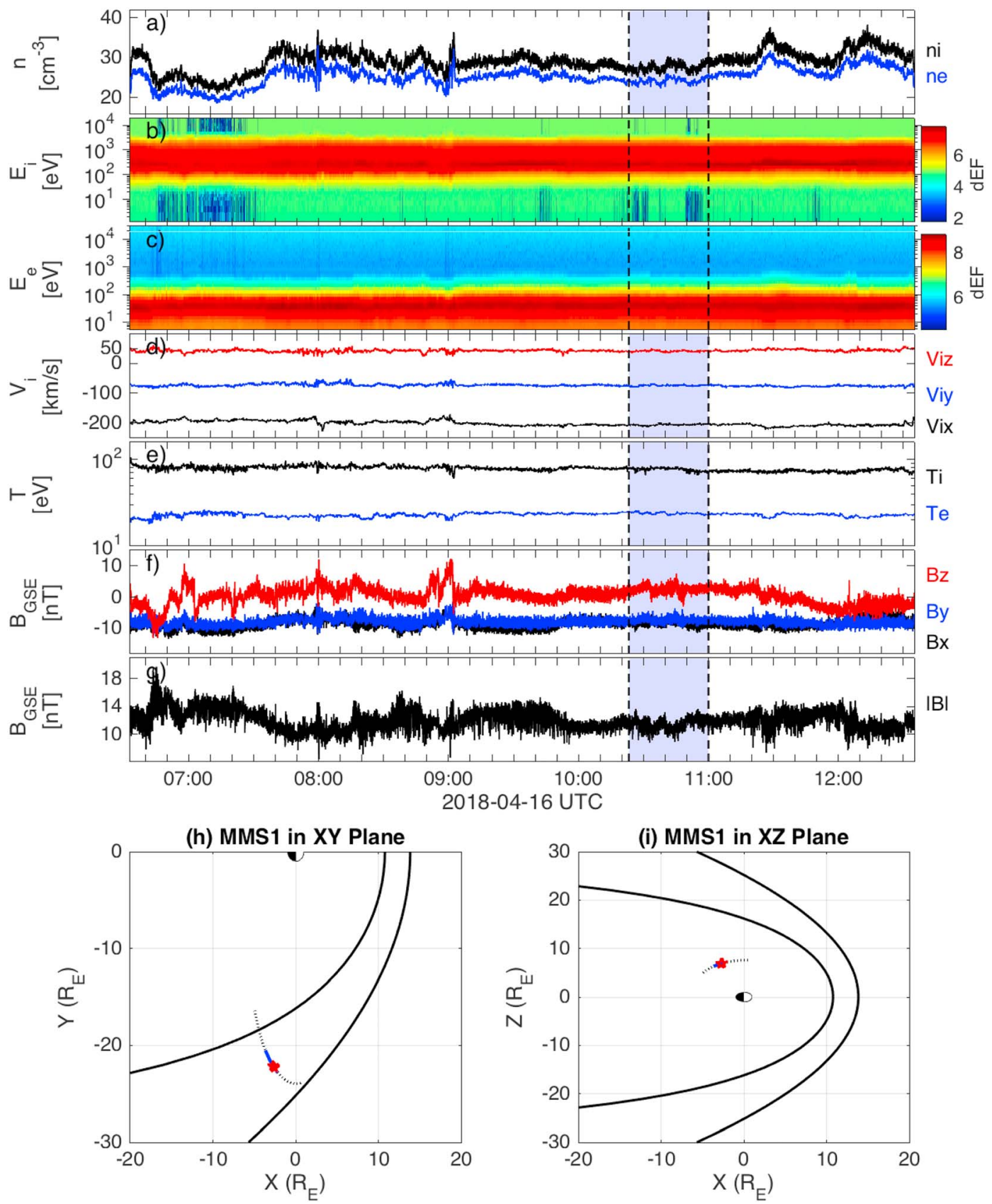

Figure 1. Overview for the plasma and magnetic field measurements from MMS1 during 06:33:00-12:35:00 on 16 April 2018. (a) The ion and electron density; (b) the ion spectrogram of differential energy flux; (c) the electron spectrogram of differential energy flux; (d) the ion bulk velocity; (e) the ion and electron temperature; (f) the magnetic field in GSE coordinates; and (g) the total magnetic field. The MMS1 trajectory in $X Y$ and $X Z$ planes in GSE coordinates is shown in panels $(\mathrm{h})$ and (i), where the dotted and dashed lines correspond to the time interval of 00:00:01-23:59:59 and 06:33:0012:35:00 on 16 April 2018. The burst mode continually operates in the time interval of 10:23:14-10:58:32 UT, labeled by two dashed lines in panels (a)-(g) and by the red stars in panels (h)-(i).

The PSDs of perpendicular magnetic field components exhibit remarkable enhancements in the range of $0.1-1 \mathrm{~Hz}$, nearly at and above the local proton cyclotron frequency $f_{\mathrm{cp}} \simeq 0.17 \mathrm{~Hz}$, whereas the PSD of the parallel magnetic field in the range of $0.1-1 \mathrm{~Hz}$ is roughly an order of magnitude lower than the perpendicular magnetic field PSDs. The PSDs of perpendicular ion and electron velocities are also enhanced in 0.1-1 Hz. Since the frequencies of these enhanced perpendicular fluctuations are smaller than the Doppler shifted frequency $f_{\lambda_{p}}=V_{i \|} / \lambda_{p} \simeq 5 \mathrm{~Hz}$ at the proton inertial length $\lambda_{p}$ (estimated from 

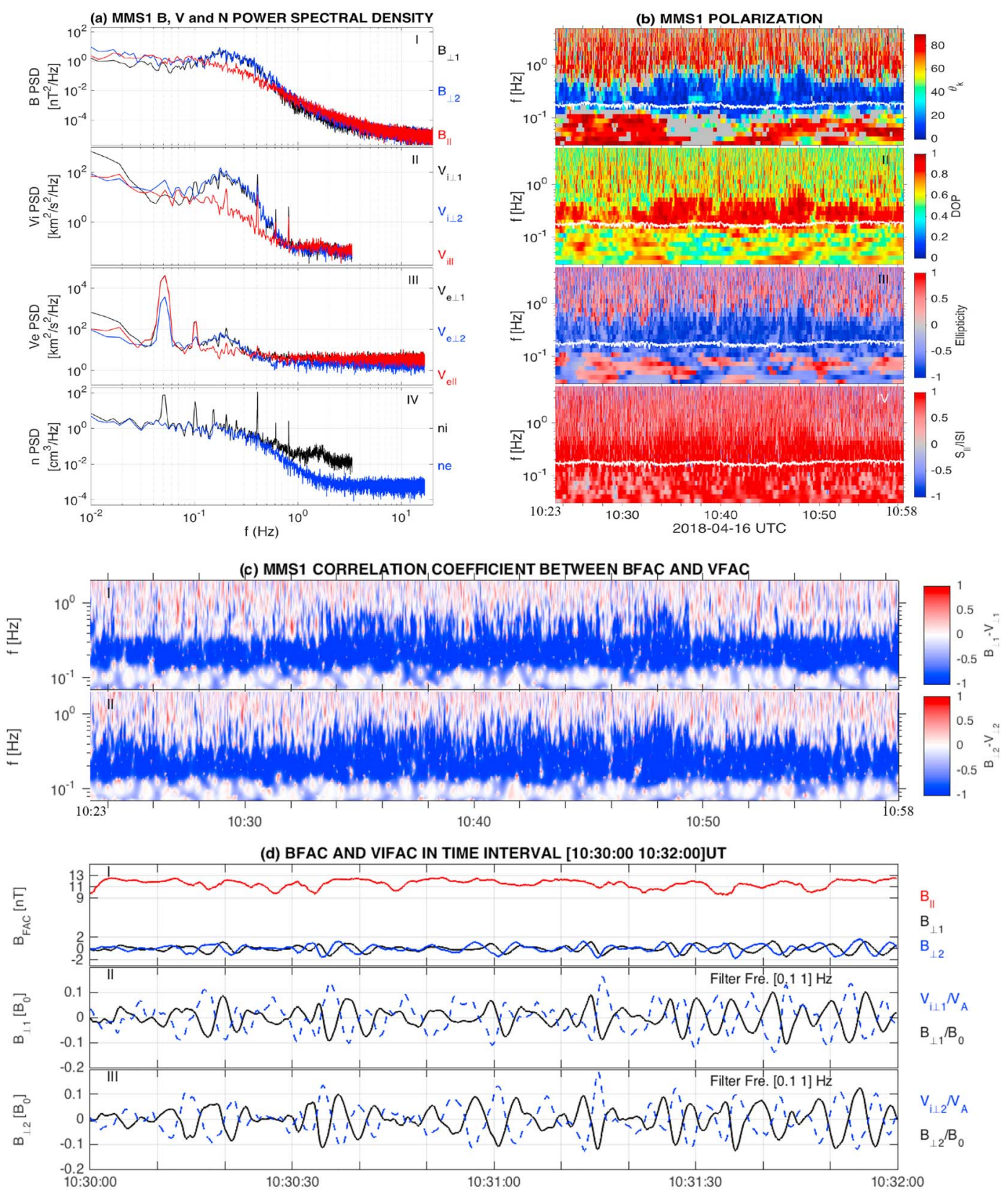

Figure 2. (a) Power spectral density (PSD) of the magnetic field (panel I), ion velocity (panel II), electron velocity (panel III), and ion and electron number density (panel IV) during 10:23:14-10:58:32 UT. (b) Polarization analysis at 0.02-5 Hz: the wave normal angle of the wave vector relative to $\mathrm{B}_{0}\left(\theta_{k}\right.$, panel I), the degree of polarization (DOP, panel II), the ellipticity (panel III), and the Poynting flux along the magnetic field $\left(\mathrm{S}_{\|} / \mathrm{IS|}\right.$, panel IV), where the white solid line represents the proton cyclotron frequency. (c) Correlation coefficients of $B_{\perp 1}$ and $V_{i \perp 1}$ (panel I) and of $B_{\perp 2}$ and $V_{i \perp 2}$ (panel II). (d) An example for the magnetic field and ion velocity in FAC during 10:30:00-10:32:00 UT, where the solid and dashed lines represent the magnetic field and ion velocity components, respectively.

$V_{i \|} \sim 205.5 \mathrm{~km} / \mathrm{s}$ and $\lambda_{p} \equiv \sqrt{m_{p} / \mu_{0} n_{p} e^{2}} \sim 40 \mathrm{~km}$ ), their scale sizes are larger than $\lambda_{p}$. The PSD of the ion number density nearly matches the PSD of the electron density at $f \sim<0.5 \mathrm{~Hz}$ and is larger than the electron density PSD at $f \sim>0.5 \mathrm{~Hz}$. We also notice a peak in ion density PSD between 1 and $2 \mathrm{~Hz}$, whereas there is no such signature in the PSDs of the magnetic field and velocity. Moreover, several spikes are found at certain frequencies $f \simeq(0.4 \mathrm{~Hz}, 0.6 \mathrm{~Hz}, 0.8 \mathrm{~Hz})$ in the $\mathrm{V}_{\mathrm{i}}$ PSD (Figure 2a-II) and at $f \simeq$ $\left(0.05 \mathrm{~Hz}, 0.1 \mathrm{~Hz}, 0.15 \mathrm{~Hz}, 0.2 \mathrm{~Hz}, 0.25 \mathrm{~Hz}\right.$ ) in the $\mathrm{V}_{\mathrm{e}}$ PSD (Figure 2a-III). The spikes at these frequencies 
are also seen in the PSD of the ion density (Figure 2a-IV). These periodic signatures may be a result of a spacecraft spin effect, and not due to a local physical process.

Figure $2 \mathrm{~b}$ presents the polarization analysis of the waves based on the singular value decomposition method (e.g., Lee \& Angelopoulos, 2014; Li et al., 2013; Santolík et al., 2003). It shows clear monochromatic wave signatures between 0.1 and $1 \mathrm{~Hz}$, and the waves have small normal angles $\left(\lesssim 20^{\circ}\right)$, strong degree of polarization $(\gtrsim 0.8)$, and left-handed ellipticity near -0.75 . These features indicate the observed waves are consistent with left-handed quasi-field aligned EMIC waves. Since the phase speeds of EMIC waves $\left(V_{\mathrm{ph}} \sim V_{A} \simeq 47.2 \mathrm{~km} / \mathrm{s}\right.$ ) are much smaller than the parallel bulk flow velocity $V_{i \|}$, the Taylor assumption, $f \simeq \mathrm{V}_{i \|} / \lambda$, can be used to estimate the wavelength $\lambda$ of EMIC waves. For $f \sim 0.2 \mathrm{~Hz}$ (corresponding to the peak in the $B_{\perp}$ PSD, Figure 2a-I), $\lambda$ is approximately $1,028 \mathrm{~km}$.

Figure $2 \mathrm{c}$ shows the correlation coefficient between $B_{\perp}$ and $V_{\mathrm{i} \perp}$ using the wavelet coherence technique (Grinsted et al., 2004). The correlation coefficient $\mathrm{CC}_{i j}$ is defined as $\mathrm{CC}_{i j}=R_{i j}^{2} \cos \left(\phi_{i j}\right)$, where $R_{i j}^{2}$ and $\phi_{i j}$ are the coherency and phase difference angle of two signals " $i$ " and " $j$ " (Grinsted et al., 2004). Here the background magnetic field is chosen as running averages of magnetic field data over $10 \mathrm{~s}$, not 1,000 s performed in Figure 2a. It can give a more precise estimation of the mean magnetic field for EMIC waves with the time scale smaller than $10 \mathrm{~s}$. Strong anticorrelation $\left(C_{B_{\perp}, V_{i \perp}} \lesssim-0.85\right)$ arises for $B_{\perp}$ and $V_{\mathrm{i} \perp}$ components in the range of 0.1-1 Hz. Furthermore, Figure 2d presents an example of the magnetic field and ion velocity during 10:30:00-10:32:00 UT. $B_{\perp 2}$ is nearly $\pi / 2$ out of phase with $B_{\perp 1}$ (Figure $2 \mathrm{~d}-\mathrm{I}$ ). The two $\mathrm{B}_{\perp}$ components in the 0.1 - to $1-\mathrm{Hz}$ range are anticorrelated with the corresponding $\mathrm{V}_{\mathrm{i} \perp}$ component, and the amplitude of the normalized magnetic field $B_{\perp} / B_{0}$ nearly equals the amplitude of the normalized ion velocity $V_{\mathrm{i} \perp} / V_{A}$ (Figures 2d-II and 2d-III). These relations between $B_{\perp}$ and $V_{\mathrm{i} \perp}$ further identify the existence of left-hand EMIC waves.

Figure 3a presents the coherence coefficients $\mathrm{CC}_{B_{\|} n}$ and $\mathrm{CC}_{B_{\|} V_{\|}}$during 10:23:14-10:58:32 UT. Since the ion density PSD contains sharp peaks likely resulting from a spacecraft spin effect, we use the electron density to calculate $\mathrm{CC}_{B_{\|} n}$. The positive $\mathrm{CC}_{B_{\|} n}$ noticeably dominates in the interval of $\sim 10: 34: 00-10: 50: 00$ UT (Figure 3a-I). This positive $\mathrm{CC}_{B_{\|} n}$ is suggested as a result of EMIC waves mixed with low-frequency cutoff mode (Lacombe et al., 1995). During the interval of 10:27:00-10:33:00 UT, the negative $\mathrm{CC}_{B_{\|} n}$ is dominant (Figure 3a-I), even at $f>1 \mathrm{~Hz}$. On the other hand, $B_{\|}$and $V_{\|}$are anticorrelated (Figure 3a-II), and a correlation coefficient $C_{B_{\|}, V_{\|}}<-0.55$ is seen in a number of filamentary regions. An example for $B_{\|}, V_{\|}$, and $n$ during 10:30:00-10:32:00 UT is presented in Figure 3b. At $f<0.1 \mathrm{~Hz}$, a mixture of anticorrelation, positive correlation, and $\pi / 2$ out of phase of $B_{\|}$with respect to $n$ is found in Figure 3b-III, whereas for $0.1 \mathrm{~Hz}<f<1 \mathrm{~Hz}$, anticorrelation is dominant in Figure $3 \mathrm{~b}-\mathrm{V} . B_{\|}$and $V_{\|}$are mostly anticorrelated, as shown in Figures 3bIV and 3b-VI. Weak positive correlation between $B_{\|}$and $V_{\|}$still arises, especially at $f>1 \mathrm{~Hz}$ (Figure 3bVI). Note that Figure $3 \mathrm{~b}$ only exhibits an example of anticorrelation of $B_{\|}$with $n$. If we choose a different time interval, positive correlation between $B_{\|}$and $n$ would appear in filamentary regions with $C_{B_{\|}, V_{i \|}}<0$ and $0.1 \mathrm{~Hz}<f<1 \mathrm{~Hz}$.

\section{Wave Excitation Mechanism: Instability Analysis}

Our observations identify large-amplitude EMIC waves and density fluctuations with scales larger than the proton inertial length. For theoretical study on their excitation mechanism, we need to analyze the ion and electron dynamics accompanying the wave. However, as shown in Figure 1a, compared to the ion density, the electron density is underestimated, $n_{e}<n_{i}$, and this is likely due to a part of low-energy electrons not being detected by the FPI electron detector whose lowest energy channel is $\sim 7 \mathrm{eV}$. Therefore, we use the ion density as the total plasma density, $n_{0}=n_{i}$. The ion $f_{i}$ and electron $f_{e}$ phase space densities are presented in Figures S1 and S2 in the supporting information. A sum of five drifting bi-Maxwellian proton (electron) components is used to fit $f_{i}\left(f_{e}\right)$. The fitted parameters are summarized in the supporting information. These fitted parameters ensure zero total charge, $\sum_{j} n_{\mathrm{pj}}=\sum_{j} n_{\mathrm{ej}}=\mathrm{n}_{0}=26.86 \mathrm{~cm}^{-3}$, and zero total current, $J_{0}=e\left(\sum_{j} n_{\mathrm{pj}} \mathbf{V}_{\mathrm{pdj}}-\sum_{j} n_{\mathrm{ej}} \mathbf{V}_{\mathrm{edj}}\right)=0$.

Using the fitted parameters and $B_{0}=11.5 \mathrm{nT}$ for modeling, we numerically solved the plasma kinetic model (Xie \& Xiao, 2016) and show the theoretical predictions in Figure 4. Here the wave vector is defined as 
(a) MMS1 CORRELATION AMONG $B_{\|}, N$, AND $v_{\|}$

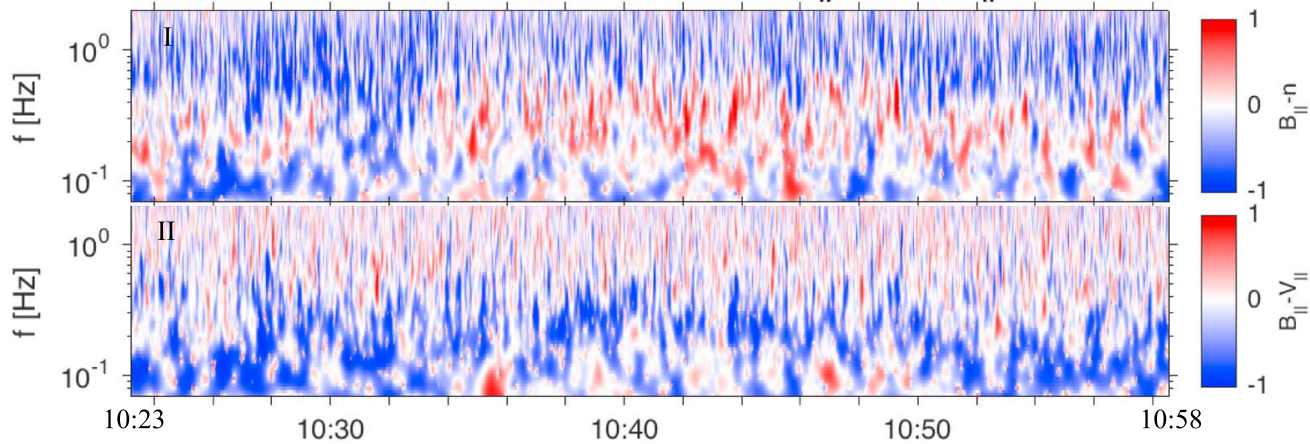

(b) $B_{\|}, \mathrm{N}$, AND $V_{\|}$IN TIME INTERVAL [10:30:00 10:32:00]UT
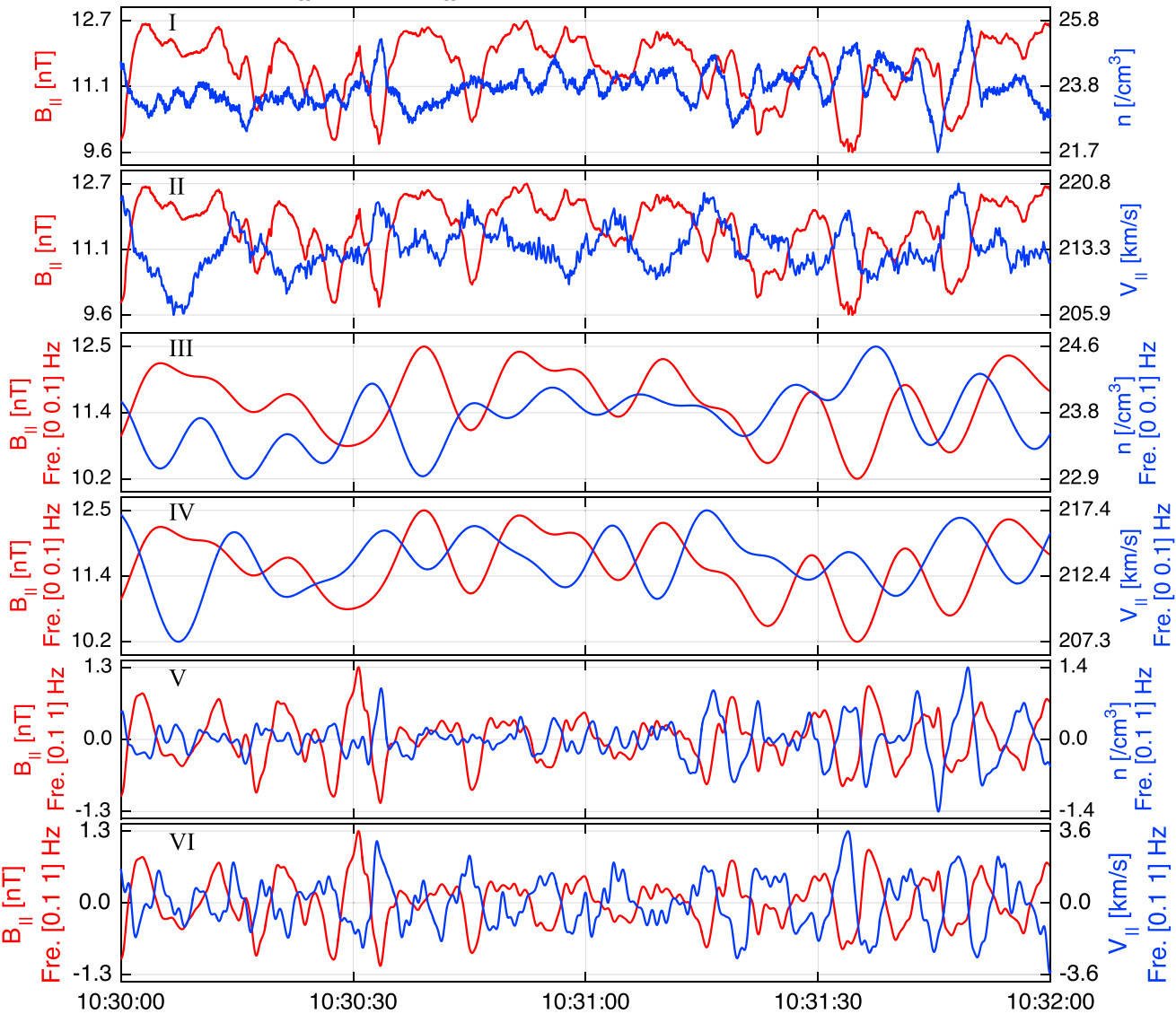

Figure 3. (a) Correlation of the parallel magnetic field $B_{\|}$to the electron density $n$ (panel I) and to the parallel ion velocity $V_{\|}$(panel II). (b) $B_{\|}, V_{\|}$, and $n$ during 10:30:00-10:32:00 UT: $B_{\|}$and $n$ (panel I), $V_{\|}$and $n$ (panel II), $B_{\|}$and $n$ filtered in the range $0-0.1 \mathrm{~Hz}$ (panel III), $V_{\|}$and $n$ filtered in $0-0.1 \mathrm{~Hz}$ (panel IV), $B_{\|}$and $n$ filtered in $0.1-1 \mathrm{~Hz}$ (panel V), and $V_{\|}$and $n$ filtered in $0.1-1 \mathrm{~Hz}$ (panel VI).

$\mathbf{k}=k_{x} \mathbf{e}_{\boldsymbol{x}}+k_{z} \mathbf{e}_{\boldsymbol{z}}$, where $\mathbf{e}_{\boldsymbol{x}} \equiv \mathbf{B}_{\mathbf{0}} \times\left(\mathbf{k} \times \mathbf{B}_{\mathbf{0}}\right) /\left|\mathbf{B}_{\mathbf{0}} \times\left(\mathbf{k} \times \mathbf{B}_{\mathbf{0}}\right)\right|$ and $\mathbf{e}_{z} \equiv \mathbf{B}_{\mathbf{0}} / B_{0}$. As shown in Figures $4 \mathrm{a}$ and $4 \mathrm{~b}$, the theory predicts two distinct instability distributions: a nearly parallel distribution at $\theta \sim<40^{\circ}$ with maximum $\gamma_{i}$ appearing at $\theta=0^{\circ}\left(\gamma_{\mathrm{i}} \simeq 0.15 f_{\text {cp }}\right.$ and $f \simeq 2.80 f_{\text {cp }}$, labeled by point "A"), and an oblique distribution at $\theta \sim>30^{\circ}$ with maximum $\gamma_{\mathrm{i}}$ appearing at $\theta \simeq 62^{\circ}\left(\gamma_{\mathrm{i}} \simeq 0.12 f_{\mathrm{cp}}\right.$, and $f \simeq 0.97 f_{\mathrm{cp}}$, labeled by point "B"). The strong excitation $\gamma_{i^{\sim}}>0.1 f_{\text {cp }}$ occurs in the wavelength range $1 / \lambda \sim 0.05 / \lambda_{p}-0.12 / \lambda_{p}$.

Figure $4 \mathrm{c}$ and $4 \mathrm{~d}$ show all unstable modes at points $\mathrm{A}$ and $\mathrm{B}$, respectively. At point $\mathrm{A}$, the strongest instability (the red line) produces the left-hand circularly polarized EMIC mode wave. The other two 

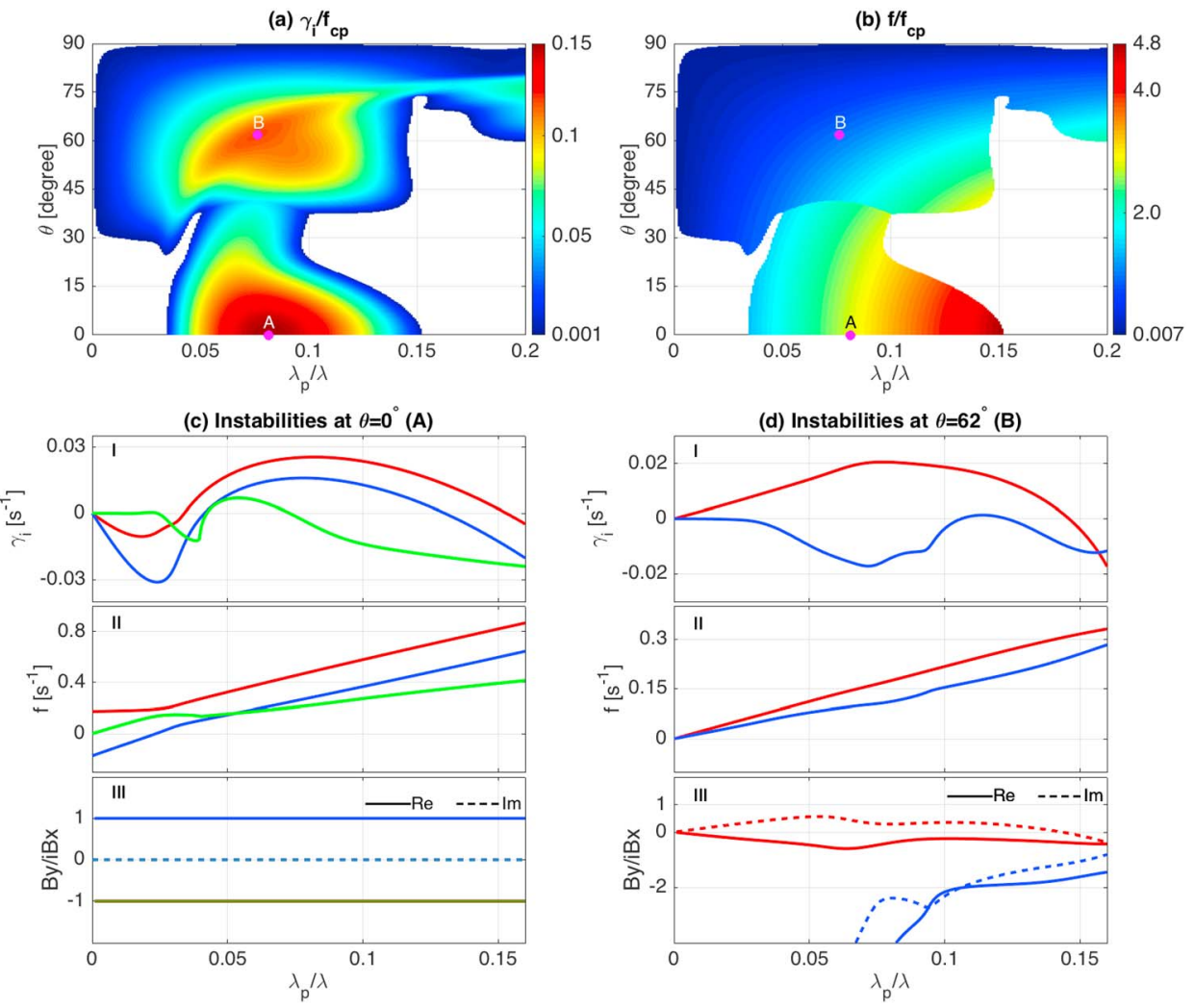

Figure 4. Linear instabilities by using the fitted plasma parameters from 10:31:00 to 10:32:00 UT. (a) Maximum growth rate $\gamma_{i}$ as functions of the wave angle $\theta$ and the wavelength $\lambda$. (b) The wave frequency $f$ at positions of $\gamma_{i}$. (c) The growth/damping rate $\gamma_{i}$, real frequency $f$, and $B_{y} / i B_{x}$ at $\theta=0^{\circ}$. (d) $\gamma_{i}, f$, and $B_{y} / i B_{x}$ at $\theta=62^{\circ}$. In panels for $B_{y} / i B_{x}$, the solid and dashed lines represent the real and imaginary part of $B_{y} / i B_{x}$. Points $\mathrm{A}$ and $\mathrm{B}$ denote the position of maximum value in the nearly parallel and oblique instability distributions. The growth rates in descending order are labeled by the red, blue, and green lines.

weak instabilities, shown by the blue and green lines, are caused by the proton anisotropic cyclotron instability and proton-proton beam instability. The proton beam-driven growing mode (the green line) is left-hand polarized, and its frequency is lower than the frequencies of unstable EMIC modes (the red line). At point $\mathrm{B}$, the strongest growth rate (the red line) exhibits a complex polarization feature, that is, $\operatorname{Re}\left(B_{y} / i B_{x}\right) \neq 0$ and $\operatorname{Im}\left(B_{y} / i B_{x}\right) \neq 0$, which cannot be simply classified into left-hand $B_{y} /$ $i B_{x}<0$ or right-hand $B_{y} / i B_{x}>0$. This instability corresponds to the mirror instability. We note that streaming protons would affect wave amplification and the dispersion relation of both EMIC and mirror instabilities. For example, they may cause asymmetry of the growth rate of unstable EMIC waves at directions of along and counter to $\mathrm{B}_{0}$ and lead to a nonzero real frequency for mirror mode waves in the plasma frame. We show these as Figures S4 and S5. We also stress that this event actually contains a small population of alpha particles $\left(n_{\alpha} \approx 0.39 \mathrm{~cm}^{-3}\right)$ as shown in Figure S6, and these particles could result in a weak instability for lower frequency EMIC waves $(f<0.1 \mathrm{~Hz})$, and slightly weaken the strongest EMIC instability (see Figure S7) compared to the corresponding instability in Figure 4.

From Figures 4 and S3-S5, we believe that the EMIC instability contributes to the observed EMIC waves. The predicted wavelengths in the region of $\gamma_{i} \sim>0.1 f_{\mathrm{cp}}$ are around $366-878 \mathrm{~km}$, approximately the observed characteristic scale. The predicted maximum growth rate $\gamma_{\text {imax }}$ at $\theta=0^{\circ}$ occurs at $f \simeq 0.49 \mathrm{~Hz}$, near the observed frequency of EMIC waves. In addition, the mirror instability occurring at $\theta=62^{\circ}$ has $\gamma_{\text {imax }}$ slightly smaller than that predicted for the EMIC instability, and the real frequency at $\gamma_{\text {imax }}$ is nearly $0.17 \mathrm{~Hz}$. The mirror instability should also be triggered under the same plasma environment, and thus, the mirror mode may contribute to density fluctuations. 


\section{Discussion and Summary}

This study presents clear observations of large-amplitude EMIC waves in the dawnside flank of the Earth's magnetosheath. Modeling shows strong temperature anisotropies of the major ion components are capable of rendering EMIC waves unstable, suggesting the waves observed by MMS were locally generated. Also, $\gamma_{i}>0.1 f_{\mathrm{cp}}$ in the EMIC instability model corresponds to the excitation of EMIC waves with normal angles smaller than $20^{\circ}$ (Figure 4a). EMIC waves propagating at $\theta \neq 0^{\circ}$ are not exactly circularly polarized, and the mixing of EMIC waves at $\theta \neq 0^{\circ}$ with the waves at $\theta=0^{\circ}$ would result in the ellipticity deviating from -1 (Denton et al., 1996).

Density fluctuations $n$ during 10:23:14-10:58:32 UT are likely caused by both EMIC and mirror mode waves. $n$ in the range of $0.1 \mathrm{~Hz}<f<1 \mathrm{~Hz}$ is both positively and negatively correlated with $B_{\|}$. Positive correlation between $n$ and $B_{\|}$is proposed to be partly contributed by nearly parallel EMIC waves (Lacombe et al., 1995). One may think of the fast magnetosonic wave for explaining this positive correlation. The fast wave is excluded due to two facts: (1) We do not detect any signal for right-handed polarized fast mode waves in the polarization analysis for electromagnetic waves with $0.1 \mathrm{~Hz}<f<1 \mathrm{~Hz}$ (Figure 2b), and (2) the predictions of linear instability do not support excitation of the fast mode. On the other hand, negative correlation of $n$ and $B_{\|}$can result from the slow magnetosonic wave and/or the mirror mode. Although negative correlation between $B_{\|}$and $n\left(V_{\|}\right)$is consistent with the theoretical predictions of the oblique slow magnetosonic wave (Zhang et al., 2018), we do not find a linear instability solution for the slow wave in the plasma kinetic model, nor do we observe a signature of the resonant condition for the slow wave being nonlinearly generated by large-amplitude EMIC waves (Hollweg, 1994; Wong \& Goldstein, 1986). Therefore, the mirror mode is the most likely explanation for negative correlation between $n$ and $B_{\|}$. Furthermore, linear kinetic theory also supports triggering of the mirror instability.

The mixing of both EMIC and mirror mode waves is also supported by previous magnetosheath observations and simulations (e.g., Hubert et al., 1998; McKean et al., 1995; Narita \& Glassmeier, 2005). Furthermore, electromagnetic and density fluctuations in our event are propagating toward the magnetopause in the flank of the magnetosheath. When EMIC waves propagate from the position $R 1$ (at 12:35:00 UT, in the outer magnetosheath) to the position $R 2$ (at 06:33:00 UT, in the inner magnetosheath), the ravel distance $L$ is $L=R 1-R 2 \sim 1.4 \times 10^{4} \mathrm{~km}$, and the travel time $T$ is $T=L / V_{0} \sim 60 \mathrm{~s}$. If EMIC waves are linearly growing, the wave amplitude at $R 2$ should be $e^{\gamma_{i} T \sim} 4-5$ times of the amplitude at $R 1$. However, observations show that the amplitude near $R 2(\sim 2 \mathrm{nT})$ is similar to the one near $R 1$. Hence, the observed waves remain saturation.

\section{Acknowledgments}

We thank the MMS team for data access and support, and the data are available at the LASPICU-Boulder website (https://lasp.colorado.edu/mms/sdc/ public/). This work was supported by the NNSFC 11673069, 41531071, and 11761131007; by NSF of Jiangsu Province under Grant BK20161617; and the Youth Innovation Promotion Association CAS. M. W. D. is supported by NNSFC 41874193 and 41574155 , NERC Grant NE/H004076/1, and by STFC in-house research grant. T. Y. W. is supported by the Marie SkłodowskaCurie Grant 665593 from the European Union's Horizon 2020 Research and Innovation Programme. J. S. H. is supported by NNSFC 41574168 and 41874200. J. S. Z thanks Huasheng Xie for helpful discussions, and the authors are grateful to two referees for useful suggestions.

\section{References}

Alexandrova, O., Mangeney, A., Maksimovic, M., Lacombe, C., Cornilleau-Wehrlin, N., Lucek, E. A., et al. (2004). Cluster observations of finite amplitude Alfvén waves and small-scale magnetic filaments downstream of a quasi-perpendicular shock. Journal of Geophysical Research, 109, A05207. https://doi.org/10.1029/2003JA010056

Anderson, B. J., Fuselier, S. A., Gary, S. P., \& Denton, R. E. (1994). Magnetic spectral signatures in the Earth's magnetosheath and plasma depletion layer. Journal of Geophysical Research, 99, 5877-5891. https://doi.org/10.1029/93JA02827

Anderson, B. J., Fuselier, S. A., \& Murr, D. (1991). Electromagnetic ion cyclotron waves observed in the plasma depletion layer. Geophysical Research Letters, 18, 1955-1958. https://doi.org/10.1029/91GL02238

Czaykowska, A., Bauer, T. M., Treumann, R. A., \& Baumjohann, W. (1998). Mirror waves downstream of the quasi-perpendicular bow shock. Journal of Geophysical Research, 103, 4747-4753. https://doi.org/10.1029/97JA03245

Czaykowska, A., Bauer, T. M., Treumann, R. A., \& Baumjohann, W. (2001). Magnetic field fluctuations across the Earth's bow shock. Annales Geophysicae, 19(3), 275-287. https://doi.org/10.5194/angeo-19-275-2001

Denton, R. E., Anderson, B. J., Ho, G., \& Hamilton, D. C. (1996). Effects of wave superposition on the polarization of electromagnetic ion cyclotron waves. Journal of Geophysical Research, 101(A11), 24,869-24,885. https://doi.org/10.1029/96JA02251

Denton, R. E., Gary, S. P., Anderson, B. J., Fuselier, S. A., \& Hudson, M. K. (1994). Low-frequency magnetic fluctuation spectra in the magnetosheath and plasma depletion layer. Journal of Geophysical Research, 99, 5893-5901. https://doi.org/10.1029/93JA02729

Ergun, R. E., Tucker, S., Westfall, J., Goodrich, K. A., Malaspina, D. M., Summers, D., et al. (2016). The axial double probe and fields signal processing for the MMS mission. Space Science Reviews, 199(1-4), 167-188. https://doi.org/10.1007/s11214-014-0115-x

Gary, S. P. (1992). The mirror and ion cyclotron anisotropy instabilities. Journal of Geophysical Research, 97, 8519-8529. https://doi.org/ 10.1029/92JA00299

Gary, S. P. (1993). Theory of Space Plasma Microinstabilities. Cambridge, UK: Cambridge University Press.

Gary, S. P., Fuselier, S. A., \& Anderson, B. J. (1993). Ion anisotropy instabilities in the magnetosheath. Journal of Geophysical Research, 98 , 1481-1488. https://doi.org/10.1029/92JA01844

Grinsted, A., Moore, J. C., \& Jevrejeva, S. (2004). Application of the cross wavelet transform and wavelet coherence to geophysical time series. Nonlinear Processes in Geophysics, 11(5/6), 561-566. https://doi.org/10.5194/npg-11-561-2004

Hollweg, J. V. (1994). Beat, modulational, and decay instabilities of a circularly polarized Alfvén wave. Journal of Geophysical Research, 99 , 23,431-23,447. https://doi.org/10.1029/94JA02185 
Hubert, D., Lacombe, C., Harvey, C. C., Moncuquet, M., Russell, C. T., \& Thomsen, M. F. (1998). Nature, properties, and origin of lowfrequency waves from an oblique shock to the inner magnetosheath. Journal of Geophysical Research, 103, 26,783-26,798. https://doi. org/10.1029/98JA01011

Hubert, D., Perche, C., Harvey, C. C., Lacombe, C., \& Russell, C. T. (1989). Observation of mirror waves downstream of a quasiperpendicular shock. Geophysical Research Letters, 16, 159-162. https://doi.org/10.1029/GL016i002p00159

Kitamura, N., Kitahara, M., Shoji, M., Miyoshi, Y., Hasegawa, H., Nakamura, S., et al. (2018). Direct measurements of two-way waveparticle energy transfer in a collisionless space plasma. Science, 361(6406), 1000-1003. https://doi.org/10.1126/science.aap8730

Lacombe, C., Belmont, G., Hubert, D., Harvey, C. C., Mangeney, A., Russell, C. T., et al. (1995). Density and magnetic field flucttuations observed by ISEE 1-2 in the quiet magnetosheath. Annales Geophysicae, 13(4), 343-357. https://doi.org/10.1007/s00585-995-0343-1

Lacombe, C., Pantellini, F. G. E., Hubert, D., Harvey, C. C., Mangeney, A., Belmont, G., \& Russell, C. T. (1992). Mirror and Alfvénic waves observed by ISEE 1-2 during crossings of the Earth's bow shock. Annales Geophysicae, 10, 772-784.

Lee, J. H., \& Angelopoulos, V. (2014). Observations and modeling of EMIC wave properties in the presence of multiple ion species as function of magnetic local time. Journal of Geophysical Research: Space Physics, 119, 8942-8970. https://doi.org/10.1002/2014JA020469

Li, W., Bortnik, J., Thorne, R. M., Cully, C. M., Chen, L., Angelopoulos, V., et al. (2013). Characteristics of the Poynting flux and wave normal vectors of whistler-mode waves observed on THEMIS. Journal of Geophysical Research: Space Physics, 118, 1461-1471. https:// doi.org/10.1002/jgra.50176

Lindqvist, P. A., Olsson, G., Torbert, R. B., King, B., Granoff, M., Rau, D., et al. (2016). The spin-plane double probe electric field instrument for MMS. Space Science Reviews, 199(1-4), 137-165. https://doi.org/10.1007/s11214-014-0116-9

Lucek, E. A., Constantinescu, D., Goldstein, M. L., Pickett, J., Pinçon, J. L., Sahraoui, F., et al. (2005). The magnetosheath. Space Science Reviews, 118(1-4), 95-152. https://doi.org/10.1007/s11214-005-3825-2

McKean, M. E., Omidi, N., \& Krauss-Varban, D. (1995). Wave and ion evolution downstream of quasi-perpendicular bow shocks. Journal of Geophysical Research, 100, 3427-3437. https://doi.org/10.1029/94JA02529

Narita, Y., \& Glassmeier, K. H. (2005). Dispersion analysis of low-frequency waves through the terrestrial bow shock. Journal of Geophysical Research, 110, A12215. https://doi.org/10.1029/2005JA011256

Pollock, C., Moore, T., Jacques, A., Burch, J., Gliese, U., Saito, Y., et al. (2016). Fast plasma investigation for Magnetospheric Multiscale. Space Science Reviews, 199(1-4), 331-406. https://doi.org/10.1007/s11214-016-0245-4

Remya, B., Tsurutani, B. T., Reddy, R. V., Lakhina, G. S., Falkowski, B. J., Echer, E., \& Glassmeier, K.-H. (2014). Large-amplitude, circularly polarized, compressive, obliquely propagating electromagnetic proton cyclotron waves throughout the Earth's magnetosheath: Low plasma $\beta$ conditions. Astrophysical Journal, 793, 6. https://doi.org/10.1088/0004-637X/793/1/6

Russell, C. T., Anderson, B. J., Baumjohann, W., Bromund, K. R., Dearborn, D., Fischer, D., et al. (2016). The Magnetospheric Multiscale magnetometers. Space Science Reviews, 199(1-4), 189-256. https://doi.org/10.1007/s11214-014-0057-3

Santolík, O., Parrot, M., \& Lefeuvre, F. (2003). Singular value decomposition methods for wave propagation analysis. Radio Science, 38(1), 1010. https://doi.org/10.1029/2000RS002523

Schwartz, S. J., Burgess, D., \& Moses, J. J. (1996). Low-frequency waves in the Earth's magnetosheath: Present status. Annales Geophysicae, 14, 1134-1150. https://doi.org/10.1007/s00585-996-1134-Z

Sckopke, N., Paschmann, G., Brinca, A. L., Carlson, C. W., \& Lühr, H. (1990). Ion thermalization in quasi-perpendicular shocks involving reflected ions. Journal of Geophysical Research, 95, 6337-6352. https://doi.org/10.1029/JA095iA05p06337

Tsurutani, B. T., Lakhina, G. S., Verkhoglyadova, O. P., Echer, E., Guarnieri, F. L., Narita, Y., \& Constantinescu, D. O. (2011). Magnetosheath and heliosheath mirror mode structures, interplanetary magnetic decreases, and linear magnetic decreases: Differences and distinguishing features. Journal of Geophysical Research, 116, A02103. https://doi.org/10.1029/2010JA015913

Tsurutani, B. T., Smith, E. J., Anderson, R. R., Ogilvie, K. W., Scudder, J. D., Baker, D. N., \& Bame, S. J. (1982). Lion roars and nonoscillatory drift mirror waves in the magnetosheath. Journal of Geophysical Research, 87, 6060-6072. https://doi.org/10.1029/JA087iA08p06060

Welch, P. (1967). The use of fast Fourier transform for the estimation of power spectra: a method based on time averaging over short, modified periodograms. IEEE Transactions on Audio and Electroacoustics, 15(2), 70-73.

Wong, H. K., \& Goldstein, M. L. (1986). Parametric instabilities of circularly polarized Alfvén waves including dispersion. Journal of Geophysical Research, 91, 5617-5628. https://doi.org/10.1029/JA091iA05p05617

Xie, H., \& Xiao, Y. (2016). PDRK: A general kinetic dispersion relation solver for magnetized plasma. Plasma Science and Technology, 18(2), 97. https://doi.org/10.1088/1009-0630/18/2/01

Young, D. T., Burch, J. L., Gomez, R. G., de Los Santos, A., Miller, G. P., Wilson, P., et al. (2016). Hot plasma composition analyzer for the magnetospheric multiscale mission. Space Science Reviews, 199(1-4), 407-470. https://doi.org/10.1007/s11214-014-0119-6

Zhang, L., He, J., Zhao, J., Yao, S., \& Feng, X. (2018). Nature of magnetic holes above ion scales: a mixture of stable slow magnetosonic and unstable mirror modes in a double-polytropic scenario? Astrophysical Journal, 864, 35. https://doi.org/10.3847/1538-4357/aad4aa

Zhao, J. S., Wang, T. Y., Dunlop, M. W., He, J. S., Dong, X. C., Wu, D. J., et al. (2018). Modulation of ion and electron pitch angle in the presence of large-amplitude, low-frequency, left-hand circularly polarized electromagnetic Waves observed by MMS. Astrophysical Journal, 867(1), 58. https://doi.org/10.3847/1538-4357/aae097

Zwan, B. J., \& Wolf, R. A. (1976). Depletion of solar wind plasma near a planetary boundary. Journal of Geophysical Research, 81, 1636-1648. https://doi.org/10.1029/JA081i010p01636 